\title{
Targeting BRAF mutants in clear-cell sarcomas of soft tissue: beyond sarcoma or melanoma classification
}

\author{
Arnaud Uguen ${ }^{1,2,3,6}$ • Pascale Marcorelles ${ }^{2,3,5}$ • Marc De Braekeleer 1,3,4
}

Received: 30 November 2015 / Accepted: 28 December 2015 /Published online: 8 January 2016

(C) Springer Science+Business Media New York 2016

Keywords Clear cell sarcoma $\cdot$ Melanoma $\cdot E W S R 1 \cdot B R A F$. Atypical melanocytic tumors

\section{To the Editor,}

With interest we read the case report by Protsenko et al. on a metastatic BRAFV600E-mutated clear cell sarcoma responding to vemurafenib anti-BRAF targeted therapy [1]. We want to point out the crucial overlapping features between a melanoma and a clear-cell sarcoma from a theranostic point of view.

Clear cell sarcoma represents about $1 \%$ of all soft tissue sarcomas with an estimated yearly incidence of less than five cases per 10 million persons. With an annual incidence of some ten cases per 100,000 persons, malignant melanoma is about 200 times more frequent than clear cell sarcoma. Clear cell sarcoma is often intimately associated with tendons and aponeuroses and occurs in the extremities, with the foot/ankle

Arnaud Uguen

arnaud.uguen@chu-brest.fr

Inserm, U1078, F-29200 Brest, France

2 Service d'anatomie et cytologie pathologiques, CHRU Brest, F-29220 Brest, France

3 Université Européenne de Bretagne, Rennes, France

4 Laboratoire de Cytogénétique et Biologie de la Reproduction, CHRU Brest, F-29220 Brest, France

5 Faculté de Médecine et des Sciences de la Santé Université de Brest, EA4685, F-29200 Brest, France

6 Department of Pathology, University Hospital Morvan, 5, Avenue Foch, 29609 Brest, France accounts for $40 \%$ of the cases. The head, the neck or the trunk regions are rarely affected. This tumor usually affects young adults in their 20s and 30s and presents as a slowly growing mass. In the report, Protsenko et al. report a very unusual clinical observation of a fast-growing tumor of the left lumbar area in a 46-year-old man.

From a pathological point of view, clear cell sarcomas and malignant melanomas share many histological and immunohistochemical features. Therefore, a melanoma can easily disguise itself as a clear cell sarcoma. The histopathological differential diagnosis between a malignant melanoma, especially a metastasis, and a clear cell sarcoma is not obvious. It is even more difficult when, as seen in many melanomas, the primary tumor remains unidentified despite of the metastatic evolution (so-called melanoma of an unknown primary origin). Differential diagnosis requires a search for rearrangements of EWSR1 (22q12) gene present in about $96 \%$ of all clearcell sarcomas (according to the last WHO classification of soft tissue tumors) and absent in melanomas. Fluorescent in situ hybridization (FISH) with a EWSRl break-apart probe allows the detection of the rearrangements of the gene independently of its partner. Reverse-transcriptase polymerase chain reaction (RT-PCR) with specific primers can be used to detect the EWSR 1/ATF1 fusion due to $\mathrm{t}(12 ; 22)(\mathrm{q} 13 ; \mathrm{q} 12)$ or the EWSR1/CREB1 fusion due to $\mathrm{t}(2 ; 22)(\mathrm{q} 32.3 ; \mathrm{q} 12)$, present in about 90 and $6 \%$ of the cases, respectively.

Protsenko et al. reported the absence of EWSR $1 / A T F 1$ and $E W S R 1 / C R E B 1$ fusions. As the method used to perform these analyses is not mentioned (presumably RT-PCR), we question whether a EWSR1 break-apart probe FISH analysis might have been an interesting method in the search of a yet unknown EWSR 1 partner. Indeed, EWSR 1 rearrangement is considered to be a hallmark of a clear-cell sarcoma and has been proven helpful in differentiating clear cell sarcomas from metastatic melanomas. On the other hand, BRAFV600E mutation 
is considered a valuable argument for a malignant melanoma instead of a clear cell sarcoma [2].

The EWSR1-non rearranged and BRAFV600E-mutated clear cell sarcoma described by Protsenko et al. highly asks for the differential diagnosis between a clear cell sarcoma of soft tissue and a melanoma of unknown origin with clear cell features. Other tumors presenting similar features have been classified as clear cell sarcomas. The existence of EWSR1rearranged and $B R A F V 600 E$-mutated tumors is a strong argument for the reality of $B R A F$ mutations in authenticable clearcell sarcomas. In addition, a particular chromosomal profile with gains of chromosomes 8 and 22 is reported in clear-cell sarcomas lacking EWSR1 rearrangement but chromosomal aberrations such as gains are also hallmarks of a malignant melanoma. Some melanocytic tumors have recently been shown to harbor EWSR1 amplification and others, particularly those with Spitzoid morphological features, are now identified as containing translocations involving oncogenes such as ALK, ROS1, MET, BRAF or RET [3]. Could clear cell sarcoma be in fact a subtype of malignant melanocytic tumor with recurrent translocation instead of a sarcoma with melanocytic differentiation? Clear-cell sarcoma was formerly called "malignant melanoma of soft parts". Classification of this tumor as a sarcoma or a melanoma is still debated.

Besides the distinction between a sarcoma and a melanoma, Protsenko et al. rightly points out the interest of treating this patient as having a "BRAF-mutated malignant clear cell tumor with melanocytic differentiation". Oncologists and pathologists need to realize the interest of searching for $B R A F$ mutations in these rare tumors of poor prognosis to allow access to targeted therapies.

Acknowledgments No funding was received for this work.

\section{Compliance with ethical standards}

Conflict of interest The authors declare no conflict of interest.

\section{References}

1. Protsenko SA, Semionova AI, Komarov YI, Aleksakhina SN, Ivantsov AO, Iyevleva AG, Imyanitov EN (2015) BRAF-mutated clear cell sarcoma is sensitive to vemurafenib treatment. Investig New Drugs 33:1136-1143

2. Hocar O, Le CA, Berissi S, Terrier P, Bonvalot S, Vanel D, Auperin A, Le Pechoux C, Bui B, Coindre JM, Robert C (2012) Clear cell sarcoma (malignant melanoma) of soft parts: a clinicopathologic study of 52 cases. Dermatol Res Pract 2012:984096

3. Wiesner T, He J, Yelensky R, Esteve-Puig R, Botton T, Yeh I, Lipson D, Otto G, Brennan K, Murali R, Garrido M, Miller VA, Ross JS, Berger MF, Sparatta A, Palmedo G, Cerroni L, Busam KJ, Kutzner H, Cronin MT, Stephens PJ, Bastian BC (2014) Kinase fusions are frequent in Spitz tumours and spitzoid melanomas. Nat Commun 5: 3116 